\title{
Entire functions uniformly bounded on balls of a Banach space
}

\author{
by \\ José M. Ansemil, Jerónimo López-Salazar and \\ SOCORRo PONTE (Madrid)
}

\begin{abstract}
Let $X$ be an infinite-dimensional complex Banach space. Very recently, several results on the existence of entire functions on $X$ bounded on a given ball $B_{1} \subset X$ and unbounded on another given ball $B_{2} \subset X$ have been obtained. In this paper we consider the problem of finding entire functions which are uniformly bounded on a collection of balls and unbounded on the balls of some other collection.
\end{abstract}

1. Introduction. Throughout the article, $X$ will denote an infinitedimensional complex Banach space and $\mathcal{H}(X)$ will be the space of all entire (holomorphic) functions on $X$. If $x \in X$ and $r>0$, then $B(x, r)$ will denote the open ball in $X$ with center $x$ and radius $r$. If $f \in \mathcal{H}(X)$ and $S \subset X$, let $\|f\|_{S}=\sup _{x \in S}|f(x)|$.

When one considers a continuous linear form or a continuous polynomial on $X$, it is well-known that both are bounded on every bounded subset of $X$. But, as a consequence of the Josefson-Nissenzweig theorem (see [4, p. 219]) that yields the existence of a sequence $\left(\varphi_{n}\right)_{n=1}^{\infty}$ of norm 1 elements in $X^{*}$ which pointwise converges to 0 , the series $\sum_{n=1}^{\infty} \varphi_{n}^{n}$ is an entire function on $X$ which is unbounded on some ball of $X$ (see [6, p. 157]). Some related theorems were obtained by Aron and Kiselman in the seventies (see [3] and [7]). The following theorem mentions several improvements of the above result which have been obtained recently.

THEOREM 1.1 .

(a) (see [2]) Given two balls $B_{0}$ and $B_{1}$ in $X$ such that $B_{1} \nsubseteq B_{0}$ and a real number $\varepsilon>0$, there is an entire function $f$ on $X$ such that

$$
\|f\|_{B_{0}}<\varepsilon \text { and }\|f\|_{B_{1}}=\infty .
$$


(b) (see [8]) Let $\mathcal{H}_{b}(X)$ be the vector subspace of $\mathcal{H}(X)$ of all entire functions of bounded type, that is, bounded on every bounded subset of $X$. Then there is an infinitely generated algebra $\mathcal{A} \subset \mathcal{H}(X)$ such that

$$
\mathcal{A} \backslash\{0\} \subset \mathcal{H}(X) \backslash \mathcal{H}_{b}(X) .
$$

(c) (see [8]) Given two balls $B_{0}$ and $B_{1}$ in $X$ such that $B_{1} \nsubseteq B_{0}$, there is an infinite-dimensional vector space $\mathcal{F}$ such that

$$
\mathcal{F} \backslash\{0\} \subset\left\{f \in \mathcal{H}(X):\|f\|_{B_{0}}<\infty \text { and }\|f\|_{B_{1}}=\infty\right\} .
$$

The purpose of this paper is to study the following problem, proposed to us by Richard Aron, which is related to Theorem 1.1(a).

Problem 1.2. Let $I$ and $J$ be two subsets of $\mathbb{N} \cup\{0\}$ such that $I \cap J=\emptyset$. Let $\left\{B_{n}: n \in I \cup J\right\}$ be a collection of balls in $X$ such that $B_{j} \nsubseteq \bigcup_{i \in I} B_{i}$ for all $j \in J$ and let $\varepsilon>0$. Does there exist a function $f \in \mathcal{H}(X)$ such that $\|f\|_{B_{i}}<\varepsilon$ for every $i \in I$ and $\|f\|_{B_{j}}=\infty$ for every $j \in J$ ?

We will give answers to that problem for different choices of the sets $I$ and $J$ and of the position and size of the balls. As we will see, this is not trivial, even when the sets $I$ and $J$ are finite. In fact, we will have to introduce new techniques, which can only be applied with some restrictions. Note that Theorem 1.1(a) solves this problem for two balls.

Remark 1.3. Problem 1.2 does not always have a solution. For instance, if we assume that $X$ is separable and we consider a dense sequence $\left(x_{i}\right)_{i=1}^{\infty}$ in $\partial B(0,1)$ and $\varepsilon>0$, then by the maximum modulus principle, there is no $f \in \mathcal{H}(X)$ such that $\|f\|_{B\left(x_{i}, 1 / 2\right)}<\varepsilon$ for every $i \in \mathbb{N}$ and $\|f\|_{B(0,1 / 2)}=\infty$.

2. The results. We start with the case of $I=\{0\}$ and $J=\mathbb{N}$.

THEOREM 2.1. Let $\left(B_{n}\right)_{n=0}^{\infty}$ be a sequence of open balls in $X$ such that $B_{j} \nsubseteq B_{0}$ for every $j \in \mathbb{N}$. For each $j$, let

$$
s_{j}=\sup \left\{\|x\|: x \in B_{j}\right\}
$$

and assume that $\lim _{j \rightarrow \infty} s_{j}=\infty$. Then, given $\varepsilon>0$, there is a function $f \in \mathcal{H}(X)$ such that

$$
\|f\|_{B_{0}}<\varepsilon \text { and }\|f\|_{B_{j}}=\infty \quad \text { for every } j \in \mathbb{N} \text {. }
$$

Proof. We can assume that $B_{0}=B\left(0, R_{0}\right)$ for some $R_{0}>0$.

Since $\lim _{j \rightarrow \infty} s_{j}=\infty$, we can rearrange the sequence $\left(B_{j}\right)_{j=1}^{\infty}$ so that

$$
s_{1} \leq s_{2} \leq \cdots .
$$

Moreover, as each $B_{j}$ is an open set, we have $\|x\|<s_{j}$ for every $x \in B_{j}$.

Let $x_{1} \in B_{1} \backslash \overline{B_{0}}$. Then

$$
R_{0}<\left\|x_{1}\right\|<s_{1},
$$


so there is $m_{1} \in \mathbb{N}$ such that $\left\|x_{1}\right\|<s_{1}-1 / m_{1}$. We define $R_{1}=s_{1}-1 / m_{1}$, which satisfies $R_{1}>R_{0}$. Since $R_{1}<s_{1} \leq s_{2}$, there is $x_{2} \in B_{2}$ such that

$$
R_{1}<\left\|x_{2}\right\|<s_{2} .
$$

Again, we take $m_{2} \in \mathbb{N}, m_{2}>m_{1}$, such that $\left\|x_{2}\right\|<s_{2}-1 / m_{2}$ and we define $R_{2}=s_{2}-1 / m_{2}$.

In this way, we get two sequences $\left(x_{j}\right)_{j=1}^{\infty} \subset X$ and $\left(R_{j}\right)_{j=0}^{\infty} \subset \mathbb{R}^{+}$with the following properties:

(a) $\left(R_{j}\right)_{j=0}^{\infty}$ is increasing and $\lim _{j \rightarrow \infty} R_{j}=\infty$ because $\lim _{j \rightarrow \infty} s_{j}=\infty$,

(b) for all $j \geq 1, x_{j} \in B_{j} \cap B\left(0, R_{j}\right)$ and $x_{j} \notin \bar{B}\left(0, R_{j-1}\right)$.

Let $r_{1}>0$ be such that $B\left(x_{1}, r_{1}\right) \subset B_{1} \cap B\left(0, R_{1}\right)$ and $B\left(x_{1}, r_{1}\right) \cap$ $B\left(0, R_{0}\right)=\emptyset$. Then by Theorem 1.1(a), there exists a function $f_{1} \in \mathcal{H}(X)$ such that

$$
\left\|f_{1}\right\|_{B\left(0, R_{0}\right)}<1 / 2 \text { and }\left\|f_{1}\right\|_{B\left(x_{1}, r_{1}\right)}=\infty .
$$

Let $r_{2}>0$ be such that $B\left(x_{2}, r_{2}\right) \subset B_{2} \cap B\left(0, R_{2}\right), B\left(x_{2}, r_{2}\right) \cap B\left(0, R_{1}\right)=\emptyset$ and $\left\|f_{1}\right\|_{B\left(x_{2}, r_{2}\right)}<\infty$. Again by Theorem 1.1(a), there exists $f_{2} \in \mathcal{H}(X)$ such that

$$
\left\|f_{2}\right\|_{B\left(0, R_{1}\right)}<1 / 2^{2} \text { and }\left\|f_{2}\right\|_{B\left(x_{2}, r_{2}\right)}=\infty .
$$

By repeating these arguments, we obtain two sequences $\left(r_{j}\right)_{j=1}^{\infty} \subset \mathbb{R}^{+}$and $\left(f_{j}\right)_{j=1}^{\infty} \subset \mathcal{H}(X)$ such that

(c) $B\left(x_{j}, r_{j}\right) \subset B_{j} \cap B\left(0, R_{j}\right)$,

(d) $B\left(x_{j}, r_{j}\right) \cap B\left(0, R_{j-1}\right)=\emptyset$,

(e) $\left\|f_{n}\right\|_{B\left(x_{j}, r_{j}\right)}<\infty$ if $1 \leq n \leq j-1$,

(f) $\left\|f_{j}\right\|_{B\left(0, R_{j-1}\right)}<1 / 2^{j}$,

(g) $\left\|f_{j}\right\|_{B\left(x_{j}, r_{j}\right)}=\infty$.

Let $K$ be a compact subset of $X$. By (a), there is $j \in \mathbb{N}$ such that $K \subset B\left(0, R_{j}\right)$. Then

$$
\sum_{n=j+1}^{\infty}\left\|f_{n}\right\|_{K} \leq \sum_{n=j+1}^{\infty}\left\|f_{n}\right\|_{B\left(0, R_{j}\right)} \leq \sum_{n=j+1}^{\infty}\left\|f_{n}\right\|_{B\left(0, R_{n-1}\right)} \leq \sum_{n=j+1}^{\infty} \frac{1}{2^{n}}<\infty .
$$

Therefore, the series $\sum_{n=1}^{\infty} f_{n}$ converges uniformly on compact subsets of $X$ and defines a holomorphic function $f$ on $X$.

The function $f$ is bounded on $B\left(0, R_{0}\right)$ :

$$
\|f\|_{B\left(0, R_{0}\right)} \leq \sum_{n=1}^{\infty}\left\|f_{n}\right\|_{B\left(0, R_{0}\right)} \leq \sum_{n=1}^{\infty}\left\|f_{n}\right\|_{B\left(0, R_{n-1}\right)} \leq \sum_{n=1}^{\infty} \frac{1}{2^{n}}<\infty .
$$


In addition, if $j \in \mathbb{N}$, then

$$
\begin{aligned}
\|f\|_{B_{j}} & \geq\left\|\sum_{n=1}^{\infty} f_{n}\right\|_{B\left(x_{j}, r_{j}\right)} \\
& \geq\left\|f_{j}\right\|_{B\left(x_{j}, r_{j}\right)}-\sum_{n=1}^{j-1}\left\|f_{n}\right\|_{B\left(x_{j}, r_{j}\right)}-\sum_{n=j+1}^{\infty}\left\|f_{n}\right\|_{B\left(x_{j}, r_{j}\right)} \\
& \geq\left\|f_{j}\right\|_{B\left(x_{j}, r_{j}\right)}-\sum_{n=1}^{j-1}\left\|f_{n}\right\|_{B\left(x_{j}, r_{j}\right)}-\sum_{n=j+1}^{\infty}\left\|f_{n}\right\|_{B\left(0, R_{n-1}\right)} .
\end{aligned}
$$

By (e), (f) and (g) we deduce that $\|f\|_{B_{j}}=\infty$. To complete the proof, it suffices to take the function $\frac{\varepsilon}{\|f\|_{B_{0}}+1} f$.

REMARK 2.2. If we consider only a finite collection of balls $\left(B_{n}\right)_{n=0}^{m}$ such that $B_{j} \nsubseteq B_{0}$ for every $j \in\{1, \ldots, m\}$, then the above proof can be stopped at step $m$. In that case, it is trivial that the function $\sum_{n=1}^{m} f_{n}$ has the properties we want.

In the proof of the next theorem we will need a result about biorthogonal systems. In [5, p. 250], Dilworth, Girardi and Johnson proved that in every infinite-dimensional Banach space $X$ there is a biorthogonal system $\left\{x_{n}, \varphi_{n}\right\}_{n=1}^{\infty}$ such that $\lim _{n \rightarrow \infty} \varphi_{n}(x)=0$ for every $x \in X,\left\|\varphi_{n}\right\|=1$ for every $n$ and $\sup \left\|x_{n}\right\|<\infty$. The proof of this fact follows from an inductive process, so we can fix a finite number of vectors $x_{1}, \ldots, x_{m+1}$ and functionals $\varphi_{1}, \ldots, \varphi_{m+1}$ and complete them with a system $\left\{x_{n}, \varphi_{n}\right\}_{n=m+2}^{\infty}$ with the properties given above:

Proposition 2.3. Given $x_{1}, \ldots, x_{m+1} \in X$ and $\varphi_{1}, \ldots, \varphi_{m+1} \in X^{*}$, there are two sequences $\left(x_{n}\right)_{n=m+2}^{\infty} \subset X$ and $\left(\varphi_{n}\right)_{n=m+2}^{\infty} \subset X^{*}$ such that

(1) $\lim _{n \rightarrow \infty} \varphi_{n}(x)=0$ for every $x \in X$,

(2) $\left\|\varphi_{n}\right\|=1$ for every $n \geq m+2$,

(3) $\sup \left\|x_{n}\right\|<\infty$,

(4) $\left\{x_{n}, \varphi_{n}\right\}_{n=m+2}^{\infty}$ is a biorthogonal system,

(5) if $1 \leq i \leq m+1$ and $n \geq m+2$, then $\varphi_{n}\left(x_{i}\right)=0$ and $\varphi_{i}\left(x_{n}\right)=0$.

Theorem 2.4. Let $\left\{B\left(x_{n}, R_{n}\right)\right\}_{n=0}^{m}$ be a finite collection of balls in $X$ such that

$$
R_{0}>\max \left\{R_{1}, \ldots, R_{m}\right\} .
$$

Then there is a function $f \in \mathcal{H}(X)$ such that

$$
\|f\|_{B\left(x_{i}, R_{i}\right)}<\infty \text { for every } i \in\{1, \ldots, m\} \text { and }\|f\|_{B\left(x_{0}, R_{0}\right)}=\infty \text {. }
$$

Proof. We can assume that $x_{0}=0$. Let $0<\varepsilon<1, \varepsilon<R_{0}$, be such that

$$
\left(R_{0}-\varepsilon\right)(1-\varepsilon)>\max \left\{R_{1}, \ldots, R_{m}\right\} \text {. }
$$


Let $Y$ be a closed hyperplane such that $\operatorname{span}\left\{x_{1}, \ldots, x_{m}\right\} \subset Y$. By Riesz's lemma, there exists $z \in X$ such that $\|z\|=1$ and $\operatorname{dist}(z, Y) \geq 1-\varepsilon$. Let

$$
x_{m+1}=\left(R_{0}-\varepsilon\right) z \in B\left(0, R_{0}\right) .
$$

This vector satisfies

$$
\begin{aligned}
\operatorname{dist}\left(x_{m+1}, \operatorname{span}\left\{x_{1}, \ldots, x_{m}\right\}\right) & \geq \operatorname{dist}\left(x_{m+1}, Y\right)=\left(R_{0}-\varepsilon\right) \operatorname{dist}(z, Y) \\
& \geq\left(R_{0}-\varepsilon\right)(1-\varepsilon)>\max \left\{R_{1}, \ldots, R_{m}\right\} .
\end{aligned}
$$

By the Hahn-Banach theorem, there is $\varphi_{m+1} \in X^{*}$ such that $\left\|\varphi_{m+1}\right\|=1$, $\varphi_{m+1}\left(x_{i}\right)=0$ if $1 \leq i \leq m$ and

$$
\varphi_{m+1}\left(x_{m+1}\right)=\operatorname{dist}\left(x_{m+1}, \operatorname{span}\left\{x_{1}, \ldots, x_{m}\right\}\right) .
$$

Let us choose arbitrary functionals $\varphi_{1}, \ldots, \varphi_{m} \in X^{*}$ and let $\left(x_{n}\right)_{n=m+2}^{\infty} \subset X$ and $\left(\varphi_{n}\right)_{n=m+2}^{\infty} \subset X^{*}$ be sequences with the properties in Proposition 2.3.

As $\sup \left\|x_{n}\right\|<\infty$, there is $r>0$ such that $x_{m+1}+r x_{n} \in B\left(0, R_{0}\right)$ for every $n$. Since $\varphi_{m+1}\left(x_{m+1}\right)>\max \left\{R_{1}, \ldots, R_{m}\right\}$, there exists $c>0$ with

$$
\max \left\{R_{1}, \ldots, R_{m}\right\}<1 / c<\varphi_{m+1}\left(x_{m+1}\right) .
$$

Then

$$
c R_{i}<1<c \varphi_{m+1}\left(x_{m+1}\right)
$$

for every $i \in\{1, \ldots, m\}$, so there is $\alpha \in \mathbb{N}$ such that $\left(c R_{i}\right)^{\alpha} R_{i}<1$ for every $i \in\{1, \ldots, m\}$ and $\left(c \varphi_{m+1}\left(x_{m+1}\right)\right)^{\alpha} r>1$.

The function

$$
f=\sum_{n=m+2}^{\infty}\left(\left(c \varphi_{m+1}\right)^{\alpha} \varphi_{n}\right)^{n}
$$

is holomorphic on $X$ since $\lim _{n \rightarrow \infty} \varphi_{n}(x)=0$ for every $x \in X$. If $1 \leq i \leq m$, then $f$ is bounded on $B\left(x_{i}, R_{i}\right)$ :

$$
\begin{aligned}
\|f\|_{B\left(x_{i}, R_{i}\right)} & =\sup _{\|x\|<R_{i}}\left|f\left(x_{i}+x\right)\right|=\sup _{\|x\|<R_{i}}\left|\sum_{n=m+2}^{\infty}\left(\left(c \varphi_{m+1}(x)\right)^{\alpha} \varphi_{n}(x)\right)^{n}\right| \\
& \leq \sum_{n=m+2}^{\infty}\left(\left(c R_{i}\right)^{\alpha} R_{i}\right)^{n}<\infty .
\end{aligned}
$$

On the other hand,

$$
\sup _{n \geq m+2}\left|f\left(x_{m+1}+r x_{n}\right)\right|=\sup _{n \geq m+2}\left(\left(c \varphi_{m+1}\left(x_{m+1}\right)\right)^{\alpha} r\right)^{n}=\infty .
$$

Thus we deduce that $\|f\|_{B\left(0, R_{0}\right)}=\infty$.

REMARK 2.5. The condition $R_{0}>\max \left\{R_{1}, \ldots, R_{m}\right\}$ in the above theorem is sometimes unnecessary, as Theorem 1.1(a) shows. 
Corollary 2.6. Let $m, n \in \mathbb{N}, n>m$, and consider two finite sequences of balls, $\left\{B\left(x_{i}, R_{i}\right)\right\}_{i=1}^{m}$ and $\left\{B\left(x_{j}, R_{j}\right)\right\}_{j=m+1}^{n}$, such that

$$
\max \left\{R_{1}, \ldots, R_{m}\right\}<\min \left\{R_{m+1}, \ldots, R_{n}\right\} .
$$

Then for every $\varepsilon>0$ there is a function $f \in \mathcal{H}(X)$ such that

$$
\|f\|_{B\left(x_{i}, R_{i}\right)}<\varepsilon \quad \text { for every } i \in\{1, \ldots, m\}
$$

and

$$
\|f\|_{B\left(x_{j}, R_{j}\right)}=\infty \quad \text { for every } j \in\{m+1, \ldots, n\} .
$$

Proof. Let us choose positive numbers $r_{m+1}, \ldots, r_{n}$ such that

$$
\max \left\{R_{1}, \ldots, R_{m}\right\}<r_{m+1}<\cdots<r_{n}<\min \left\{R_{m+1}, \ldots, R_{n}\right\} .
$$

By Theorem 2.4, there exists $f_{m+1} \in \mathcal{H}(X)$ such that $\left\|f_{m+1}\right\|_{B\left(x_{i}, R_{i}\right)}<\infty$ for every $i \in\{1, \ldots, m\}$ and $\left\|f_{m+1}\right\|_{B\left(x_{m+1}, r_{m+1}\right)}=\infty$. We have to consider two different cases:

(1) If $\left\|f_{m+1}\right\|_{B\left(x_{m+2}, r_{m+2}\right)}=\infty$, then let $f_{m+2}=f_{m+1}$.

(2) If $\left\|f_{m+1}\right\|_{B\left(x_{m+2}, r_{m+2}\right)}<\infty$, then, by Theorem 2.4, there exists a function $g_{m+1} \in \mathcal{H}(X)$ such that $\left\|g_{m+1}\right\|_{B\left(x_{i}, R_{i}\right)}<\infty$ for $1 \leq i \leq m$, $\left\|g_{m+1}\right\|_{B\left(x_{m+1}, r_{m+1}\right)}<\infty$ and $\left\|g_{m+1}\right\|_{B\left(x_{m+2}, r_{m+2}\right)}=\infty$. Let $f_{m+2}=$ $f_{m+1}+g_{m+1}$.

In both cases, $f_{m+2}$ is an entire function such that

$$
\begin{aligned}
& \left\|f_{m+2}\right\|_{B\left(x_{i}, R_{i}\right)}<\infty \quad \text { for every } i \in\{1, \ldots, m\}, \\
& \left\|f_{m+2}\right\|_{B\left(x_{m+1}, R_{m+1}\right)} \geq\left\|f_{m+2}\right\|_{B\left(x_{m+1}, r_{m+1}\right)}=\infty, \\
& \left\|f_{m+2}\right\|_{B\left(x_{m+2}, R_{m+2}\right)} \geq\left\|f_{m+2}\right\|_{B\left(x_{m+2}, r_{m+2}\right)}=\infty .
\end{aligned}
$$

The proof follows by recurrence. Finally, we take the function $\frac{\varepsilon}{C+1} f_{n}$, where $C=\max _{1 \leq i \leq m}\left\|f_{n}\right\|_{B\left(x_{i}, R_{i}\right)}$.

Theorem 2.7. Let $X$ be a Banach space with a Schauder basis $\left(e_{n}\right)_{n=1}^{\infty}$ such that

$$
0<\inf \left\|e_{n}\right\| \leq \sup \left\|e_{n}\right\|<\infty .
$$

Let $\left(\varphi_{n}\right)_{n=1}^{\infty} \subset X^{*}$ be the sequence of coefficient functionals associated to the basis and let $M=\sup \left\|\varphi_{n}\right\|$. If $J \subset \mathbb{N}, R_{j}>0$ for every $j \in J$ and $\varepsilon>0$, then there exists $f \in \mathcal{H}(X)$ such that

$$
\|f\|_{B(0,1 / M)}<\varepsilon, \quad\|f\|_{B\left(e_{i}, 1 / M\right)}<\varepsilon \quad \text { for every } i \in \mathbb{N} \backslash J
$$

and

$$
\|f\|_{B\left(e_{j}, R_{j}\right)}=\infty \quad \text { for every } j \in J
$$

Proof. First of all, note that $M<\infty$ because inf $\left\|e_{n}\right\|>0$ (see [9, p. 20]). For each $j \in J$, let $t_{j} \in \mathbb{R}$ be such that

$$
1<t_{j}<1+\frac{R_{j}}{\left\|e_{j}\right\|} .
$$


Then $t_{j} e_{j} \in B\left(e_{j}, R_{j}\right)$. As $\sup \left\|e_{n}\right\|<\infty$, there is $r_{j}>0$ such that $t_{j} e_{j}+$ $r_{j} e_{n} \in B\left(e_{j}, R_{j}\right)$ for every $n \in \mathbb{N}$. Since $t_{j}>1$, there is $\alpha_{j} \in \mathbb{N}$ such that $\frac{1}{3} t_{j}^{\alpha_{j}} r_{j}>1$.

Let $K$ be a compact subset of $X$. As inf $\left\|e_{n}\right\|>0$, we have $\lim _{n \rightarrow \infty} \varphi_{n}(x)$ $=0$ for all $x \in X$ (see [9, p. 21]). Therefore, $\lim _{n \rightarrow \infty}\left\|\varphi_{n}\right\|_{K}=0$ as well, so there exists $n_{0} \in \mathbb{N}$ such that $\left\|\varphi_{n}\right\|_{K} \leq 1$ for all $n \geq n_{0}$. Moreover, there is $n_{1} \in \mathbb{N}, n_{1}>n_{0}$, such that

$$
\left(\sup _{j \in J, j \leq n_{0}-1}\left\|\varphi_{j}^{\alpha_{j}}\right\|_{K}\right) \cdot\left\|\varphi_{n}\right\|_{K} \leq 1
$$

for all $n \geq n_{1}$. Thus,

$$
\begin{aligned}
\sum_{j \in J} \sum_{n=j+1}^{\infty} & \left\|\left(\frac{1}{3} \varphi_{j}^{\alpha_{j}} \varphi_{n}\right)^{n}\right\|_{K} \\
& \leq \sum_{\substack{j \in J \\
j \leq n_{0}-1}} \sum_{n=j+1}^{n_{1}-1}\left\|\frac{1}{3} \varphi_{j}^{\alpha_{j}} \varphi_{n}\right\|_{K}^{n}+\sum_{\substack{j \in J \\
j \leq n_{0}-1}} \sum_{n=n_{1}}^{\infty} \frac{1}{3^{n}}+\sum_{j=n_{0}}^{\infty} \sum_{n=j+1}^{\infty} \frac{1}{3^{n}}<\infty .
\end{aligned}
$$

This implies that the series

$$
\sum_{j \in J} \sum_{n=j+1}^{\infty}\left(\frac{1}{3} \varphi_{j}^{\alpha_{j}} \varphi_{n}\right)^{n}
$$

converges uniformly on compact subsets of $X$. Consequently, it defines an entire function $f$ on $X$.

This function is bounded on $B(0,1 / M)$ :

$$
\|f\|_{B(0,1 / M)} \leq \sum_{j \in J} \sum_{n=j+1}^{\infty}\left(\frac{1}{3}\left(\left\|\varphi_{j}\right\| \frac{1}{M}\right)^{\alpha_{j}}\left\|\varphi_{n}\right\| \frac{1}{M}\right)^{n} \leq \sum_{j=1}^{\infty} \sum_{n=j+1}^{\infty} \frac{1}{3^{n}}<\infty .
$$

Let $i \in \mathbb{N} \backslash J$ and $x \in X,\|x\|<1 / M$. We have

$$
\begin{aligned}
\left|f\left(e_{i}+x\right)\right| & \leq \sum_{j \in J} \sum_{n=j+1}^{\infty}\left(\frac{\left|\varphi_{j}(x)\right|^{\alpha_{j}} \cdot\left|\varphi_{n}\left(e_{i}\right)+\varphi_{n}(x)\right|}{3}\right)^{n} \\
& \leq \sum_{j \in J} \sum_{n=j+1}^{\infty}\left(\frac{\left|\varphi_{n}\left(e_{i}\right)\right|+\left|\varphi_{n}(x)\right|}{3}\right)^{n} \leq \sum_{j=1}^{\infty} \sum_{n=j+1}^{\infty}\left(\frac{2}{3}\right)^{n}<\infty .
\end{aligned}
$$

Therefore,

$$
\|f\|_{B\left(e_{i}, 1 / M\right)}=\sup _{\|x\|<1 / M}\left|f\left(e_{i}+x\right)\right| \leq \sum_{j=1}^{\infty} \sum_{n=j+1}^{\infty}\left(\frac{2}{3}\right)^{n}<\infty .
$$

If we now fix $j \in J$, then

$$
\|f\|_{B\left(e_{j}, R_{j}\right)} \geq \sup _{n \geq j+1}\left|f\left(t_{j} e_{j}+r_{j} e_{n}\right)\right|=\sup _{n \geq j+1}\left(\frac{1}{3} t_{j}^{\alpha_{j}} r_{j}\right)^{n}=\infty .
$$


To complete the proof of the theorem, we take the function $\frac{\varepsilon}{C+1} f$, where

$$
C=\sum_{j=1}^{\infty} \sum_{n=j+1}^{\infty}\left(\frac{2}{3}\right)^{n}<\infty .
$$

Acknowledgements. The second author has been supported by Universidad Complutense de Madrid, grant BE45/08.

\section{References}

[1] J. M. Ansemil, R. M. Aron and S. Ponte, Representation of spaces of entire functions on Banach spaces, Publ. Res. Inst. Math. Sci. 45 (2009), 383-391.

[2] - , - - Behavior of entire functions on balls in a Banach space, Indag. Math. (N.S.) 20 (2009), 483-489.

[3] R. M. Aron, Entire functions of unbounded type on a Banach space, Boll. Un. Mat. Ital. 9 (1974), 28-31.

[4] J. Diestel, Sequences and Series in Banach Spaces, Springer, New York, 1984.

[5] S. J. Dilworth, M. Girardi and W. B. Johnson, Geometry of Banach spaces and biorthogonal systems, Studia Math. 140 (2000), 243-271.

[6] S. Dineen, Complex Analysis on Infinite Dimensional Spaces, Springer Monogr. Math., Springer, London, 1999.

[7] C. O. Kiselman, On the radius of convergence of an entire function in a normed space, Ann. Polon. Math. 33 (1976), 39-55.

[8] J. López-Salazar, Vector spaces of entire functions of unbounded type, Proc. Amer. Math. Soc. 139 (2011), 1347-1360.

[9] I. Singer, Bases in Banach Spaces I, Springer, Berlin, 1970. 